\begin{tabular}{|c|l|}
\hline Title & Creating human germ cells for unmet reproductive needs \\
\hline Author(s) & Ishii, Tetsuya; Pera, Renee A Reijo \\
\hline Citation & $\begin{array}{l}\text { Nature Biotechnology, 34(5), 470-473 } \\
\text { https://doi.org/40.1038/hbt.3559 }\end{array}$ \\
\hline Issue Date & 2016-05-06 \\
\hline Doc URL & http://hdl.handle.net/2115/63474 \\
\hline Type & article (author version) \\
\hline File Information & NBT.3559 (Ishii\&Pera).pdf \\
\hline
\end{tabular}

Instructions for use 
Published online 06 May 2016

\section{Creating human germ cells for unmet reproductive needs}

To the editor:

Current assisted reproductive technology (ART), such as in vitro fertilization (IVF) and intracytoplasmic sperm injection (ICSI), depends on the premise that both partners produce fertile eggs or sperm (gametes). As yet, there is no ART treatment for patients with few or no gametes, unless donor gametes are used. Ongoing research suggests that autologous female or male germ cells may be regenerated from somatic cells by controlling cell fate; if so, there is a possibility that ART might aid infertile couples and even same-sex couples in the future. Before such ART treatments using induced germ cells can be considered in the clinical context, however, a great many questions concerning the safety and efficacy of such procedures must be answered. Moreover, profound ethical and social concerns will arise if such induced germ cells are needlessly generated, cryopreserved and used for reproductive purposes in clinics without defining their appropriate roles in ART. As a response to the increasing feasibility of inducing germ cells from human pluripotent stem cells, we discuss below the current technical challenges to creating induced human germ cells and explore some of the ethical, legal and social issues associated with their use in clinical practice.

Recent work has shown that mouse oocytes as well as spermatozoa can be differentiated from induced pluripotent stem cells (iPSCs) and embryonic stem cells (ESCs) via primordial germ cell-like cells (PGCLCs), resulting in the birth of healthy 
offspring $^{1,2}$. Furthermore, human germ cell induction research has also made rapid advances, including the generation of haploid, round spermatid(-like) cells from iPSCs and/or $\mathrm{ESCs}^{3-7}$.

Three major approaches have been used to induce fertile germ cells from somatic cells using stem cell technology (Fig. 1). The first makes use of the current primary methodology of stepwise differentiation of human iPSCs (generated from a patient's somatic cells) to germ cells in vitro (Fig. 1a). To date, directed differentiation of human iPSCs under the existence of bone morphogenetic proteins, retinoic acid and subsequent treatment (Forskolin, human leukemia inhibitory factor, basic fibroblast growth factor, and R115866), or mouse spermatogonial stem cell (SSC) culture conditions has generated primordial germ cells (PGCs $)^{4,8}, \mathrm{SSCs}^{9}$, spermatocytes ${ }^{5,9}$ and haploid, round spermatid(-like) cells expressing ACROSIN ${ }^{5,9}$, despite a low induction efficiency. However, fertilizing oocytes with such immature male germ cells would result in poor embryonic development ${ }^{10}$. Moreover, although a combined differentiation protocol using both in vitro and in vivo systems induced fertile oocytes from mouse iPSCs ${ }^{2}$, in vitro generation of human female germ cells has not been manifestly demonstrated.

With the advent of genome-editing technology, we can expect human germ cell induction research to advance rapidly. For example, genome editing has been used to induce PGCs from human pluripotent stem cells in vitro ${ }^{11,12}$. Using gene editing in germline-competent human ESC lines, SOX17 has been demonstrated to be a key regulator of PGC-like cell fate ${ }^{11}$. In addition, PGC-like cells can be differentiated from genetically edited human iPSCs, which display primed pluripotency, not naïve potency, via incipient mesoderm-like cells ${ }^{12}$. Of particular note is the recent finding that human 
iPSCs transplanted directly into murine seminiferous tubules not only differentiate extensively to germ-cell-like cells with morphology indistinguishable from that of fetal germ cells, but also express PGC-specific proteins ${ }^{13}$. This xenotransplantation experiment suggests that culture conditions mimicking the stem cell niche in the fetal testis or ovary can effectively direct human iPSCs to germ cell differentiation in vitro. Park et al. ${ }^{8}$ have also improved PGC generation using a co-culture system with human fetal gonadal cells. Moreover, Bucay et al. ${ }^{14}$ have reported that PGC generation from human ESCs was accompanied by the development of Sertoli-like support cells, suggesting the potential utility of support cells in germ cell induction ${ }^{14}$. These findings indicate that the creation of increasingly elaborate co-culture systems, including gonadal tissue cells, has the potential to enhance the efficiency of human germ cell induction in vitro.

Although the evidence is compelling for spermatogonial stem cells, controversy remains about the existence of oogonial stem cells ${ }^{15,16}$. If oogonial stem cells can be isolated in a reproducible manner and are sufficiently characterized, the possibility of carrying out genome editing for research use may also lead to insights that ultimately could enhance female germ cell induction in vitro for clinical applications.

A second approach for germ cell generation (Fig. 1b) involves the overexpression of key genes associated with the development of germ cells. Overexpression of genes, including DAZL, DAZ, BOULE, and VASA has facilitated entrance in meiosis, resulting in the generation of haploid, round spermatid(-like) cells from human iPSCs and/or ESCs ${ }^{3,4,6}$. Moreover, a recent mouse experiment demonstrated that simultaneous overexpression of three transcription factors, Blimpl, Prdm14 and Tfap2c, efficiently differentiate epiblast-like cells to PGCs, without 
cytokines ${ }^{17}$. These findings suggest the overexpression of genes potentially induces more advanced germ cells or bona fide spermatozoa from human iPSCs if an optimized combination of key factors is identified.

A final approach for germ cell induction involves the use of somatic cell nuclear transfer to human ESCs (Fig. 1c) ${ }^{18}$. A major challenge associated with germ cells induced from cloned ESCs is the high likelihood of heteroplasmy due to carried-over mitochondria in the karyoplast. Such heteroplasmy may unbalance evolutionarily-optimized interactions between nuclear DNA and mitochondrial DNA ${ }^{19}$ with potentially deleterious biological and pathological consequences for the offspring's health. But as in the ART of mitochondrial donation, where the lack of abnormalities observed in adult non-human primates born following mitochondrial donation was cited, in part, as evidence for the technique's safety in the scientific review reported to the UK's Human Fertilisation and Embryology Authority last year ${ }^{20}$, experiments to study the offspring of larger animals could similarly be carried out to establish the safety of induced germ cells. But even if these concerns can be addressed, this third approach appears the least feasible of the induced germ cell approaches.

Whatever method is used, germ cell induction involves reversal of the Weismann barrier, wherein hereditary information moves only from germ cells to somatic cells ${ }^{21}$. The implications of reversing this process not only for ensuring the developmental potential of induced germ cells but also for assuring the health of resulting offspring over an entire life time should be carefully considered. Notably, some studies on the genetic integrity of human iPSCs suggest a tendency to develop changes in nuclear DNA and/or mitochondrial DNA in addition to chromosomal aberrations during reprogramming and/or subsequent culture ${ }^{22}$. Moreover, although 
human iPSCs derived from dermal fibroblasts have already been used in a clinical trial for retinal regeneration ${ }^{23}$, the somatic cell type of origin must be contemplated in terms of its clinical availability and cumulative burden of de novo mutations ${ }^{24}$.

With this in mind, it will be necessary to take precautions against the genetic instability of iPSCs used to induce germ cells, regardless of the establishment method used. Clearly, although intergenerational monitoring of mouse offspring derived from induced germ cells will provide valuable insight into the safety of this technology over an offspring's lifetime, additional studies using other animal species, particularly non-human primates, will be needed before clinical application is contemplated.

Quite apart from the technological hurdles that face the application of human germ cell technology to clinical practice, these procedures raise several ethical concerns $^{25}$. First, human fetal ovarian or testicular tissue will likely be required for effectively inducing oocytes or spermatozoa in vitro ${ }^{8}$. When the fetal tissues are derived from elective abortions, some might feel that the germ cell generation comes at the expense of other human lives. However, fetal tissues can be ethically procured if informed consent is obtained from patients who undergo the surgery for a clear medical reason (e.g., ectopic pregnancy). In such cases, the disclosure of information regarding the materials and methods is important.

In addition to these concerns, ART using germ cells derived from cloned human ESCs poses two other serious ethical challenges (Fig. 1c). First and foremost is the concern that the creation of human ESCs would involve the destruction of human embryos. In addition, the establishment of a cloned ESC would require oocyte retrieval with medication and hormone injections that might impose distress or potentially could lead to ovarian hyperstimulation syndrome ${ }^{26}$ in healthy oocyte donors. Clearly, 
approaches using iPSCs obtained under informed consent from an adult as a starting point (Fig. 1a,b) pose fewer ethical concerns than somatic cell nuclear transfer to human ES cells (Fig. 1c).

Then, there is the question of medical need. The potential benefits of induced germ cells are likely found in self-use by a couple because it is their germ cells that would be regenerated to have a genetically related child. To illustrate some of the scenarios $^{25-31}$, we discuss four relevant cases in Box 1.

The first reproductive use of induced spermatozoa would occur in a clinical trial. In ART treatments, informed consent is provided by prospective parent(s). On an informed consent, the potential risks associated with ART using induced germ cells include embryonic arrest, implantation failure, miscarriage and childbirth with congenital anomalies. Parental consent for the reproductive use of such germ cells is justifiable if the safety can be considered to be equivalent to that of conventional ART treatments according to the results of preclinical research ${ }^{27}$.

Beyond the above technical, ethical and social issues, the clinical use of induced germ cells must take place in an appropriate legal environment. According to the European Society of Human Reproduction and Embryology, the most ART-active countries in the world are Japan and the USA ${ }^{28}$. Twenty-three European countries have legislation on $\mathrm{ART}^{29}$, whereas the USA and Japan have few federal or national laws or guidelines directly pertaining to $\mathrm{ART}^{30}$. In general, ART is, without strict regulations, conducted as a type of advanced medicine in the USA and in Japan. The US Food and Drug Administration (FDA; Rockville, MD) regulates gamete donation with a view to preventing the transmission of infectious diseases, and this is a minimum requirement for all ART procedures. But given the reproductive use of induced germ cells appears to 
be legal both in the USA and in Japan, the elaboration of clear regulations that guide socially acceptable uses of induced germ cells should be a priority. Paradoxically, while oversight of clinical practice remains lax in the United States, the Dickey-Wicker amendment (H.R. 2880, Sec. 128) prohibits the use of appropriated funds for the creation of human embryos for research purposes or for research in which human embryos are destroyed.

It is important to point out that it will be necessary for ART practitioners using induced germ cells to provide evidence that no genetic alternation has occurred during the process because in many countries, germline genetic modification is banned; indeed, a recent survey of regulations suggests at least 29 countries (including Japan but not the USA) ban germline gene modification for reproductive purposes ${ }^{30}$. In the United States, the FDA regulates germline gene modification for safety reasons ${ }^{30}$ and the US National Institutes of Health (NIH; Bethesda, MD) Recombinant DNA Advisory Committee states in its Guidelines for Research Involving Recombinant or Synthetic Nucleic Acid Molecules, that it will not, at present, entertain proposals for germline gene alterations ${ }^{30}$. Similarly, Japan bans clinical research that intentionally conducts, or may result in, the genetic modification of human germ cells or embryos under the Guidelines of Clinical Research Regarding Gene Therapy ${ }^{30}$.

Overall, autologous induced germ cells from autologous iPSCs could benefit many couples who at present do not benefit from ART. Although a caring, nurturing and loving environment is important for family building, in reality most parents would prefer to have their own genetically related child rather than one created from an unrelated gamete donor ${ }^{27}$. Given that a mere single perfectly induced spermatozoon would be sufficient for starting ICSI if a fertile oocyte were available, we believe that a 
likely first clinical trial using induced germ cells would evaluate induced spermatozoa in ART for male cancer survivors. Nevertheless, before such a trial can take place, many ethical, legal and social issues remain to be addressed. We contend that now is the time to start discussions about how induced germ cells will be adopted into clinical ART practice.

\section{Acknowledgements}

We thank Professor H. T. Greely (Stanford University) for reading the initial version. We also acknowledge helpful comments by Dr. M. Ohnuki (Ludwig-Maximilians University Munich). This work was supported by JSPS KAKENHI Grant Number $26460586(T I)$.

Tetsuya Ishii ${ }^{1}$ \& Renee. A. Reijo Pera ${ }^{2}$

1Office of Health and Safety, Hokkaido University, Sapporo 060-0808, Japan.

2Department of Cell Biology and Neurosciences and Department of Chemistry and Biochemistry, Montana State University, Bozeman, MT 59717-2460, USA.

E-mail: tishii@general.hokudai.ac.jp

1 Hayashi, K., Ohta, H., Kurimoto, K., Aramaki, S. \& Saitou, M. Reconstitution of the mouse germ cell specification pathway in culture by pluripotent stem cells. Cell 146, 519-532, doi:10.1016/j.cell.2011.06.052 (2011).

2 Hayashi, K. et al. Offspring from oocytes derived from in vitro primordial germ cell-like cells in mice. Science (New York, N.Y.) 338, 971-975, 
doi:10.1126/science.1226889 (2012).

Kee, K., Angeles, V. T., Flores, M., Nguyen, H. N. \& Reijo Pera, R. A. Human DAZL, DAZ and BOULE genes modulate primordial germ-cell and haploid gamete formation. Nature 462, 222-225, doi:10.1038/nature08562 (2009).

Panula, S. et al. Human germ cell differentiation from fetal- and adult-derived induced pluripotent stem cells. Human molecular genetics 20, 752-762, doi:10.1093/hmg/ddq520 (2011).

$5 \quad$ Eguizabal, C. et al. Complete meiosis from human induced pluripotent stem cells. Stem cells (Dayton, Ohio) 29, 1186-1195, doi:10.1002/stem.672 (2011).

6 Medrano, J. V., Ramathal, C., Nguyen, H. N., Simon, C. \& Reijo Pera, R. A. Divergent RNA-binding proteins, DAZL and VASA, induce meiotic progression in human germ cells derived in vitro. Stem cells (Dayton, Ohio) 30, 441-451, doi:10.1002/stem.1012 (2012).

$7 \quad$ Easley, C. A. t. et al. Direct differentiation of human pluripotent stem cells into haploid spermatogenic cells. Cell reports 2, 440-446, doi:10.1016/j.celrep.2012.07.015 (2012).

8 Park, T. S. et al. Derivation of primordial germ cells from human embryonic and induced pluripotent stem cells is significantly improved by coculture with human fetal gonadal cells. Stem cells (Dayton, Ohio) 27, 783-795, doi:10.1002/stem.13 (2009).

9 Easley, Charles A. I. V. et al. Direct Differentiation of Human Pluripotent Stem Cells into Haploid Spermatogenic Cells. Cell Reports 2, 440-446, doi:10.1016/j.celrep.2012.07.015 (2012).

10 Levran, D., Nahum, H., Farhi, J. \& Weissman, A. Poor outcome with round spermatid injection in azoospermic patients with maturation arrest. Fertility and sterility 74, 443-449 (2000).

11 Irie, N. et al. SOX17 is a critical specifier of human primordial germ cell fate. Cell 160, 253-268, doi:10.1016/j.cell.2014.12.013 (2015).

12 Sasaki, K. et al. Robust In Vitro Induction of Human Germ Cell Fate from Pluripotent Stem Cells. Cell stem cell, doi:10.1016/j.stem.2015.06.014 (2015).

13 Ramathal, C. et al. Fate of iPSCs derived from azoospermic and fertile men following xenotransplantation to murine seminiferous tubules. Cell reports 7, 1284-1297, doi:10.1016/j.celrep.2014.03.067 (2014).

14 Bucay, N. et al. A novel approach for the derivation of putative primordial germ cells and sertoli cells from human embryonic stem cells. Stem cells (Dayton, Ohio) 27, 68-77, doi:10.1634/stemcells.2007-1018 (2009). 
15 White, Y. A. et al. Oocyte formation by mitotically active germ cells purified from ovaries of reproductive-age women. Nature medicine 18, 413-421, doi:10.1038/nm.2669 (2012).

16 Zhang, H. et al. Experimental evidence showing that no mitotically active female germline progenitors exist in postnatal mouse ovaries. Proceedings of the National Academy of Sciences of the United States of America 109, 12580-12585, doi:10.1073/pnas.1206600109 (2012).

17 Nakaki, F. et al. Induction of mouse germ-cell fate by transcription factors in vitro. Nature 501, 222-226, doi:10.1038/nature12417 (2013).

18 Yamada, M. et al. Human oocytes reprogram adult somatic nuclei of a type 1 diabetic to diploid pluripotent stem cells. Nature 510, 533-536, doi:10.1038/nature13287 (2014).

19 Reinhardt, K., Dowling, D. K. \& Morrow, E. H. Medicine. Mitochondrial replacement, evolution, and the clinic. Science (New York, N.Y.) 341, 1345-1346, doi:10.1126/science.1237146 (2013).

20 Greenfield, A. Third scientific review of the safety and efficacy of methods to avoid mitochondrial disease through assisted conception: 2014 update. http://www.hfea.gov.uk/docs/Third Mitochondrial replacement scientific review.pdf (2014).

21 Weismann, A., Newton Parker, W. and Ro"nnfeldt, H. Germ-Plasm, a theory of heredity. . (Scribner's., 1893).

22 Ronen, D. \& Benvenisty, N. Genomic stability in reprogramming. Current opinion in genetics \& development 22, 444-449, doi:10.1016/j.gde.2012.09.003 (2012).

23 Garber, K. RIKEN suspends first clinical trial involving induced pluripotent stem cells. Nature biotechnology 33, 890-891, doi:10.1038/nbt0915-890 (2015).

24 Lynch, M. Rate, molecular spectrum, and consequences of human mutation. Proceedings of the National Academy of Sciences of the United States of America 107, 961-968, doi:10.1073/pnas.0912629107 (2010).

25 Ishii, T., Pera, R. A. \& Greely, H. T. Ethical and legal issues arising in research on inducing human germ cells from pluripotent stem cells. Cell stem cell 13, 145-148, doi:10.1016/j.stem.2013.07.005 (2013).

26 Orvieto, R. Ovarian hyperstimulation syndrome- an optimal solution for an unresolved enigma. Journal of ovarian research 6, 77, doi:10.1186/1757-2215-6-77 (2013).

27 Ishii, T. Potential impact of human mitochondrial replacement on global policy regarding germline gene modification. Reproductive biomedicine online 29, 150-155, 
doi:10.1016/j.rbmo.2014.04.001 (2014).

ART fact

sheet

(July

2014).

http://www.eshre.eu/Guidelines-and-Legal/ART-fact-sheet.aspx (2014).

29 Busardo, F. P. \& Gulino, M. The evolution of legislation in the field of Medically Assisted Reproduction and embryo stem cell research in European union members. 2014, 307160, doi:10.1155/2014/307160 (2014).

30 Ishii, T. Germ line genome editing in clinics: the approaches, objectives and global society. Briefings in functional genomics, doi:10.1093/bfgp/elv053 (2015).

31 Palacios-Gonzalez, C., Harris, J. \& Testa, G. Multiplex parenting: IVG and the generations to come. Journal of medical ethics 40, 752-758, doi:10.1136/medethics-2013-101810 (2014).

32 Trujillo, J. P. S., J. Savior siblings and Fanconi anemia: analysis of success rates from the family's perspective. Genet Med., doi:10.1038/gim.2014.206 (2015).

33 Strong, K., Kerridge, I. \& Little, M. Savior siblings, parenting and the moral valorization of children. Bioethics 28, 187-193, doi:10.1111/j.1467-8519.2012.02001.x (2014).

34 Lee, E., Illingworth, P., Wilton, L. \& Chambers, G. M. The clinical effectiveness of preimplantation genetic diagnosis for aneuploidy in all 24 chromosomes (PGD-A): systematic review. Human reproduction (Oxford, England) 30, 473-483, doi:10.1093/humrep/deu303 (2015).

35 Schattman, G. L. A healthy dose of reality for the egg-freezing party. Fertility and sterility, doi:10.1016/j.fertnstert.2015.12.001 (2015).

36 Quinn, G. P., Zebrack, B. J., Sehovic, I., Bowman, M. L. \& Vadaparampil, S. T. Adoption and cancer survivors: Findings from a learning activity for oncology nurses. Cancer, doi:10.1002/cncr.29322 (2015).

37 Tournaye, H., Dohle, G. R. \& Barratt, C. L. Fertility preservation in men with cancer. Lancet (London, England) 384, 1295-1301, doi:10.1016/s0140-6736(14)60495-5 (2014).

\section{Box 1. Induced human germ cells in the clinic}

One use case for induced human germ cells would involve a same-sex couple. Self-use of induced oocytes derived from XY cells or induced spermatozoa from XX cells by a same-sex couple is conceivable ${ }^{31}$; however, it remains only a remote scientific possibility ${ }^{25}$. Apart from the technical challenges, one ethical issue for such an 
application is that children resulting from such a procedure might suffer distress and stigma as a result of being born as a result of an unnatural biological process.

A second use case of the clinical availability of induced oocytes would be couples looking to create a 'savior sibling' who could provide a compatible transplant for an existing sick child. Such a savoir sibling would be identified by human leukocyte antigen typing via preimplantation genetic diagnosis (PGD); such an approach is sometimes attempted with traditional ART but is relatively inefficient ${ }^{32}$. That said, human oocyte induction currently remains a work in progress as discussed above, and creating a savior sibling may be ethically controversial because it implies a moral decision by parents to favor the welfare of an existing original sick child over that of the new savior offspring ${ }^{33}$.

A third use case is the adoption of induced oocytes to assist females who have experienced recurrent ART failures due to aneuploidies associated with age-related oocyte senescence, but do not wish to use donor gametes. Although PGD to test for aneuploidy is already in practice to improve the ART success rate, its clinical effectiveness is currently unclear ${ }^{34}$. However, as mentioned above, human oocyte induction requires further research before such procedures could be contemplated. What's more, induced oocytes would also be unlikely to benefit all females of advanced age. As women get older, implantation and ability to carry a viable pregnancy may be compromised ${ }^{35}$.

A last application is infertile survivors of malignancies. Recent studies have reported that $46 \%$ of male survivors and $16 \%$ of female survivors of childhood cancer manifest infertility ${ }^{36}$. One of the major reasons is germ-cell loss due to chemotherapy and/or radiation therapy ${ }^{37}$. Although for male cancer survivors, sperm cryopreservation 
is an effective method to safeguard spermatozoa, not all male patients who are offered this method bank sperm because of stress, severe illness or sexual inexperience ${ }^{37}$; for such individuals, induced spermatozoa may provide a means of ART. For prepubertal boys, cryopreservation and subsequent transplantation of testicular tissue could restore the survivor's fertility in the future ${ }^{37}$. But ART using induced spermatozoa may be a potential reproductive option for them after understanding other options (uses of donor sperm and adoption) in addition to the risks associated with in vitro gametogenesis. 

Figure 1. Three potential approaches for inducing fertile germ cells by stem cell technology. Chr; chromosomal, ESCs; embryonic stem cells, iPSCs; induced pluripotent stem cells, PGCs; primordial germ cells, SCs; stem cells, SSCs; spermatogonial stem cells. 

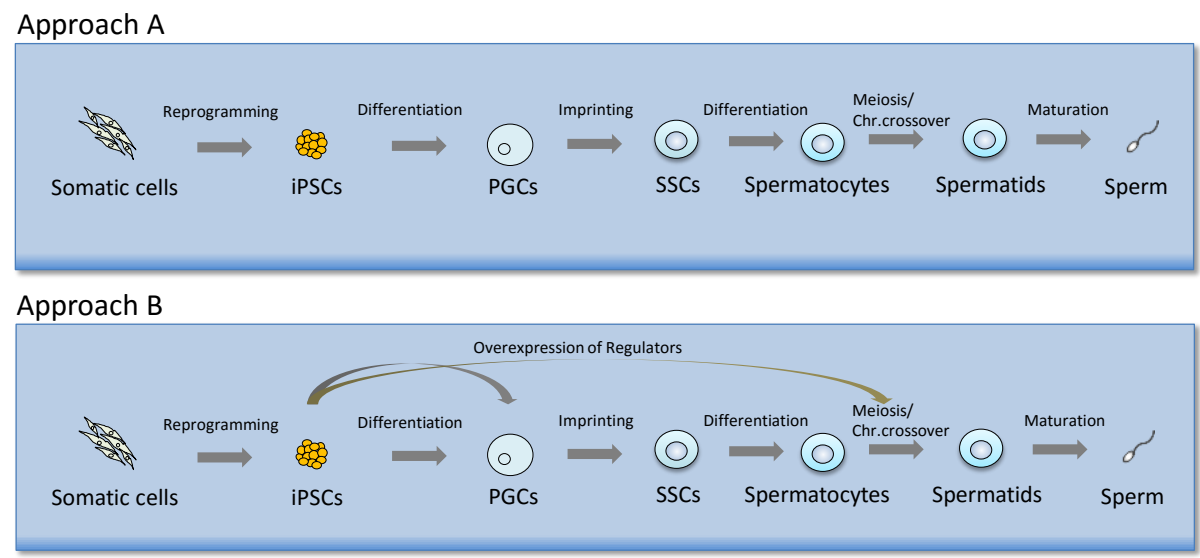

Approach C

Somatic cells

Fig 1. 